\title{
A LOCALISATION PRINCIPLE FOR QUADRATIC SPACES OVER LAURENT EXTENSIONS
}

\author{
RAMAN PARIMALA AND PARVIN SINCLAIR
}

\begin{abstract}
We prove here that the localisation principle holds for anisotropic quadratic spaces over $R\left[T, T^{-1}\right]$, where $R$ is an integral domain in which 2 is invertible. We also give an example of an isotropic quadratic space over $R\left[T, T^{-1}\right]$ for which the localisation principle does not hold.
\end{abstract}

1. Introduction. Swan, in [5], gives an example to show that Quillen's localisation principle does not hold in general for projective modules over Laurent extensions. The aim of this note is to show that the localisation principle does hold for anisotropic quadratic spaces over $R\left[T, T^{-1}\right]$, where $R$ is an integral domain in which 2 is invertible. On the other hand, using Swan's example, we exhibit an isotropic (in fact hyperbolic) quadratic space over $R\left[T, T^{-1}\right]$ which is locally and stably extended but not extended from $R$.

2. Localisation principle for anisotropic quadratic spaces. A quadratic space $(P, q)$ over a ring $R$ is called anisotropic if, for $x \in P, q(x)=0 \Rightarrow x=0$. In the sequel, we shall denote a quadratic space $(P, q)$ by $q$, suppressing the underlying module $P$.

LEMMA 2.1. Let $q$ be an anisotropic space over a domain $R$ in which 2 is invertible. Then, the natural injection $O_{R}(q) \rightarrow O_{R\left[T, T^{-1}\right]}\left(q \otimes_{R} R\left[T, T^{-1}\right]\right)$ is bijective.

Proof. Since $q \otimes_{R} R[T]$ is anisotropic modulo $T$ and $\bigcap_{n \geqslant 0} T^{n} \cdot R[T]=(0)$, using [2, Proposition 1.1], one sees that

$$
O_{R[T]}\left(q \otimes_{R} R[T]\right)=O_{R\left[T, T^{-1}\right]}\left(q \otimes_{R} R\left[T, T^{-1}\right]\right) .
$$

Let $\alpha \in O_{R[T]}\left(q \otimes_{R} R[T]\right)$ and let $\bar{\alpha}$ denote its reduction modulo $T$. Let $K$ denote the quotient field of $R$. Since $q \otimes_{R} K$ is diagonalisable and anisotropic, using [4, Lemma 2.2], it follows that $\alpha \otimes_{R[T]} K[T]=\bar{\alpha} \otimes_{R} K[T]$. Since the canonical map

$$
O_{R[T]}\left(q \otimes_{R} R[T]\right) \rightarrow O_{K[T]}\left(q \otimes_{R} K[T]\right)
$$

is injective, $\alpha=\bar{\alpha} \otimes_{R} R[T]$, and this proves the lemma.

THEOREM 2.2. Let $R$ be a domain in which 2 is invertible. Let $q$ be an anisotropic quadratic space over $R\left[T, T^{-1}\right]$. If $q \otimes_{R} R_{\mathfrak{p}}$ is extended from $R_{\mathfrak{p}}$ for every prime ideal $\mathfrak{p}$ of $R$, then $q$ is extended from $R$.

Received by the editors February 10, 1982 and, in revised form, February 16, 1983.

1980 Mathematics Subject Classification. Primary 13C10, $10 \mathrm{C} 05$.

Key words and phrases. Laurent extensions, anisotropic, quadratic spaces, localisation principle. 
Proof. Let $S=\left\{a \in R \mid q \otimes_{R} R_{a}\right.$ is extended from $\left.R_{a}\right\}$. We show that $S$ is an ideal of $R$, and since, by the hypothesis, $S$ is not contained in any maximal ideal of $R$, it follows that $S=R$ and $q$ is extended from $R$. If $a \in R, b \in S$, clearly $a \cdot b \in S$ and, therefore, to show that $S$ is an ideal, it suffices to show that for $a, b \in S, a+b \in S$. Replacing $R$ by $R_{a+b}$, it is enough to prove the following lemma.

LEMMA 2.3. Let $R$ be a domain in which 2 is invertible and let $a, b \in R$ such that $a R+b R=R$. If $q$ is an anisotropic quadratic space over $R\left[T, T^{-1}\right]$ with $q \otimes_{R} R_{a}$ and $q \otimes_{R} R_{b}$ extended from $R_{a}$ and $R_{b}$, respectively, then $q$ is extended from $R$.

Proof. Let 'bar' denote the reduction modulo $(T-1)$ and let $K$ be the quotient field of $R$. Since $q$ is anisotropic, $q^{\prime}=q \otimes_{R\left[T, T^{-1}\right]} K\left[T, T^{-1}\right]$ is anisotropic and hence, $\bar{q}^{\prime}=\bar{q} \otimes_{R} K$ is anisotropic. Hence, $\bar{q}$ is anisotropic. Let

$$
\phi: q \otimes_{R\left[T, T^{-1}\right]} R_{a}\left[T, T^{-1}\right] \stackrel{\sim}{\rightarrow} \bar{q} \otimes_{R} R_{a}\left[T, T^{-1}\right]
$$

and

$$
\psi: q \otimes_{R\left[T, T^{-1}\right]} R_{b}\left[T, T^{-1}\right] \stackrel{\sim}{\rightarrow} \bar{q} \otimes_{R} R_{b}\left[T, T^{-1}\right]
$$

be isometries. We may assume, by modifying $\phi$ and $\psi$ if necessary, that $\bar{\phi}=\bar{\psi}=$ Identity. Let $\tilde{\phi}, \tilde{\psi}$ denote the extensions of $\phi$ and $\psi$ to $R_{a b}\left[T, T^{-1}\right]$. Then $\tilde{\psi} \circ(\tilde{\phi})^{-1}$ belongs to the orthogonal group $O_{R_{a b}\left[T, T^{-1}\right]}(\bar{q})$ and $\bar{\psi} \circ \tilde{\phi}^{-1}=$ Identity. Since $\bar{q}$ is anisotropic over $R_{a b}$, by Lemma 2.1 it follows that $O_{R_{a b}\left[T, T^{-1}\right]}(\bar{q})=O_{R_{a b}}(\bar{q})$. Hence, $\tilde{\psi} \circ \tilde{\phi}^{-1}$ is independent of $T$, and $\tilde{\psi} \circ \tilde{\phi}^{-1}=$ Identity. The isometries $\phi$ and $\psi$ are defined over $R_{a}\left[T, T^{-1}\right]$ and $R_{a}\left[T, T^{-1}\right]$, respectively, which coincide over the intersection $R_{a b}\left[T, T^{-1}\right]$. Since $R a+R b=R$, it follows that $\phi$ and $\psi$ define an isometry $q \stackrel{\sim}{\rightarrow} \bar{q} \otimes_{R} R\left[T, T^{-1}\right]$ over $R\left[T, T^{-1}\right]$ and $q$ is extended from $R$.

3. A counterexample. We show that the localisation principle fails for isotropic quadratic spaces over Laurent extensions. Let

$$
R=\mathbf{C}\left[X_{0}, X_{1}, X_{2}, X_{3}, X_{4}\right] /\left\langle X_{0}^{2}+X_{1}^{2}+X_{2}^{2}+X_{3}^{2}+X_{4}^{2}-1\right\rangle
$$

be the coordinate ring of the complex 4-sphere. Let $A=R\left[T, T^{-1}\right]$ and $P$ be the projective $A$-module defined by the split exact sequence $0 \rightarrow P \rightarrow A^{3} \rightarrow A \rightarrow 0$, corresponding to the unimodular row

$$
\left(1-\frac{(1-T)}{2}\left(1-X_{0}\right), \frac{(1-T)}{2}\left(X_{1}+i X_{2}\right), \frac{(1-T)}{2}\left(X_{3}+i X_{4}\right)\right) \text {. }
$$

Then (see [5]):

(1) $P$ is stably free, in fact, $P \oplus A \stackrel{\sim}{\rightarrow} A^{3}$.

(2) $P \otimes_{R} R_{\mathfrak{p}}$ is free for every prime ideal $\mathfrak{p}$ of $R$.

(3) $P$ is not extended from $R$.

Proposition 3.1. Let $P$ be as above. Then the hyperbolic space $H(P)$ over $A$, is stably extended from $R$, locally extended from $R_{\mathfrak{p}}$ for every prime ideal $\mathfrak{p}$ of $R$, but not extended from $R$. 
Proof. By (1), $H(P) \oplus H(A) \stackrel{\sim}{\rightarrow} H\left(A^{3}\right)$ so that $H(P)$ is stably extended from $R$. By (2), $H(P) \otimes_{A} R_{\mathfrak{p}}\left[T, T^{-1}\right]$ is extended from $R_{\mathfrak{p}}$ for every prime ideal $\mathfrak{p}$ of $R$. We now show that $H(P)$ is not extended from $R$. Suppose $H(P)$ is extended from $R$. Since $P /(T-1) P$ is free, $H(P)$ is extended from $H\left(R^{2}\right)$ i.e. $H(P) \stackrel{\sim}{\rightarrow} H\left(R^{2}\right)$ $\otimes_{R} A \stackrel{\sim}{\rightarrow} H\left(A^{2}\right)$. This implies that $P$ has a unimolular element and hence $P$ itself is free. This is a contradiction to (3).

Remark 3.2. Since rank $P=2$ and $\Lambda^{2} P$ is free, we have $P \stackrel{\sim}{\rightarrow} P^{*}$. Since $P \oplus$ $A \stackrel{\sim}{\rightarrow} A^{3}$, in view of a result of Gabel cited in [3], $P \oplus P$ is free. Hence, the underlying module $P \oplus P^{*}$ of $H(P)$ is free. We thus have a nonsingular symmetric matrix over $R\left[T, T^{-1}\right]$ which is locally and stably extended from $R$, but not extended from $R$.

\section{REFERENCES}

1. H: Bass, Algebraic K-theory, Benjamin, New York, 1968.

2. M. A. Knus, R. Parimala and R. Sridharan, Non-free projective modules over $\mathbf{H}[X, Y]$ and stable bundles over $\mathbf{P}_{2}(\mathbf{C})$, Invent. Math. 65 (1981), 13-27.

3. T. Y. Lam, Series summation of stably free modules, Quart. J. Math. Oxford Ser. (2) 27 (1976), 37-46.

4. R. Parimala and R. Sridharan, A local global principle for quadratic forms over polynomial rings, J. Algebra 74 (1982), 264-269.

5. R. G. Swan, Projective modules over Laurent polynomial rings, Trans. Amer. Math. Soc. 237 (1978), $111-120$.

School of Mathematics, TATa Institute of Fundamental Research, Bombay 400005, India 